\title{
Case of HER2-Enriched Multifocal Breast Cancer Associated with Pregnancy
}

\author{
Suman Khanal', Yogendra P Singh ${ }^{2}$, Shreya Shrivastav ${ }^{3}$ \\ ${ }^{1}$ Department of Surgical Oncology, BP Koirala Memorial Cancer Hospital, ${ }^{2}$ Department of Gl and General Surgery, \\ Maharajgunj Medical Campus, Tribhuvan University Teaching Hospital, Maharajgunj, Kathmandu ${ }^{3}$ Department \\ of Pathology, Maharajgunj Medical Campus, Tribhuvan University Teaching Hospital, Maharajgunj, Kathmandu
}

\section{Corresponding author:}

\section{Suman Khanal, MBBS, MS}

Department of Surgical Oncology, BP Koirala Memorial Cancer Hospital

Email:sumandoc@iom.edu.np

Submitted : November 5, 2019

Accepted : December 6, 2019

\begin{abstract}
Pregnancy-associated breast cancer is less common and often presents in advanced stage. Management is similar to non-pregnant cases with trimester-specific considerations. In this case report, we present a case of worrisome mother with locally advanced multifocal pregnancy-associated breast cancer who was so much concerned about effects of neoadjuvant chemotherapy on fetus leading to its delayed initiation, and show why targeted therapy is still not within reach in a developing economy like Nepal. Short term data of chemotherapy though reveal relative safety, long term data are lacking. This may be a hindrance in counselling, when patients are more anxious and worrisome.
\end{abstract}

Keywords: Breast cancer, chemotherapy, HER2-neu, pregnancy

\section{INTRODUCTION}

$\mathrm{B}$ reast cancer is the most common cancer in pregnancy. ${ }^{1}$ Breast cancer that is diagnosed during pregnancy or within one year after delivery is categorized as pregnancy-associated breast cancer (PABC). PABCs usually present at advanced stage and whether it is due to rapid growth during pregnancy or diagnostic delay due to mimicking normal physiological changes is unclear. In this report, we present a case of multifocal locally advanced PABC who was very concerned about the effects of chemotherapy in the fetus leading to delay in its initiation. Targeted therapy is indicated in HER2 patients with distinct survival benefits. ${ }^{2}$ But in a developing country like Nepal with a very low gross domestic product (GDP) per capita (nominal) in whole of South Asia, the cost of it is disproportionately high compared with income. ${ }^{3}$ And hence, is within reach of few fortunate people. This case highlights the need to reduce the cost of such therapy in one of the poorest regions of the world, in such a common cancer among women. This work is reported in line with SCARE criteria. ${ }^{4}$

\section{CASE PRESENTATION}

A 35 years-old female presented at 34 weeks of pregnancy with diffuse swelling in right breast for six months. The swelling was initially located in inner upper quadrant which gradually increased to involve whole of the breast. The patient also complained of nipple retraction for 3 months. There was no history of pain, nipple discharge, trauma, significant weight loss. There was no history of similar illness in first and second-degree relatives. The patient was a past smoker with 10 pack-years of smoking history. The patient had two children, younger one being 5 years old. There was no shortness of breath. She did not have pain in the chest, back or lower limbs. She attained menarche at 16 years of age and had history of use of injectable progesterone (Depo-Provera) for 2 years in the past.

On examination, the right breast was diffusely enlarged with $12 \times 12 \mathrm{~cm}$ hard lump occupying all the quadrants with nipple retraction (Fig 1). On palpation, the surface of the mass was uneven and there was mobile axillary lymphadenopathy. There were no abnormalities noted in contralateral breast 


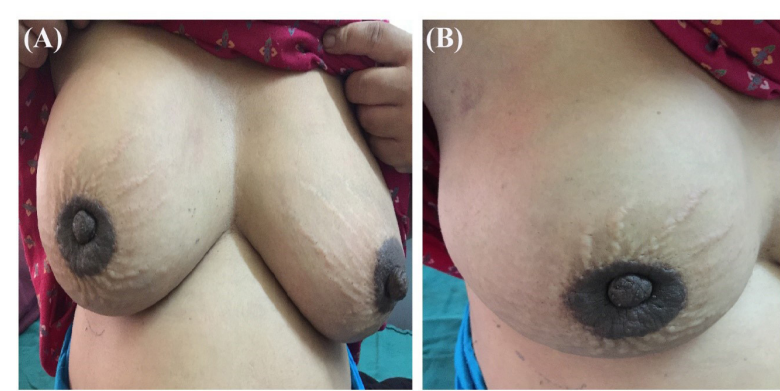

Figure 1. (A) Showing diffusely enlarged breast with elevated and retracted nipple (B) Showing small bruise in axilla (FNAC site), the site of axillary lymphadenopathy

and axilla. Solid organs in the abdomen were not palpable with absence of ascites.

Trucut biopsy of lump showed grade 2 invasive ductal carcinoma with HER2-enriched molecular subtype (estrogen receptor (ER)/progesterone (PR) negative and HER2-neu 3+) (Fig 2(A-C)) while FNAC from enlarged axillary lymph nodes also showed tumor deposits. After discussion with the medical oncologist and the patient, she was planned for neoadjuvant chemotherapy (NAC). However, she wished to deliver first instead of chemotherapy. She delivered child by caesarean section at 36 weeks. She was started on chemotherapy 4 weeks after delivery and received 3 cycles of doxorubicin, cyclophosphamide and 5-fluorouracil (FU) regimen. After 3 weeks of chemotherapy, she underwent modified radical mastectomy (MRM).

The excised specimen showed multifocal tumor, the largest one measuring $51 \mathrm{~mm}$ with grade 2 invasive ductal carcinoma, no special type (NST) histology. There was only partial pathological response with $70 \%$ residual invasive tumor, extensive desmoplasia with lymphovascular invasion but no perineural invasion (Fig 2(D)). Metastatic deposits were seen in 16 out of 24 lymph nodes retrieved from axilla with final staging of YT3N3a (Stage IIIC on AJCC seventh edition). All margins were free of tumor. Post-operatively she received additional three cycles of adjuvant chemotherapy and $50 \mathrm{~Gy}$ radiation therapy. She could not afford Trastuzumab. She was doing fine at 12 months follow up after surgery (Fig 3).

\section{DISCUSSION}

PABCs are the most common cancers in pregnancy with 1:3000 incidence with mean age of diagnosis from 32 to 38 years. ${ }^{1}$ The incidence is increasing in West due to delayed childbearing. They are often diagnosed at advanced stage and this could be one reason of the relatively poor outcome. ${ }^{5-8}$ Due to its rarity, management guideline is not as robust for non-PABCs. So general treatment principles that are applicable in non-PABCs are also applied for PABCs with few trimester-specific modifications. ${ }^{9}$ NCCN recommends chemotherapy be avoided in first trimester due to increased risk of miscarriage, stillbirth and birth defects ${ }^{10}$, and radiation be postponed altogether till after delivery. ${ }^{9}$ Surgery can be performed in all trimesters of pregnancy with the use of modern anesthesia techniques without significant risk to fetus. Hormonal therapies like Tamoxifen are not recommended in pregnancy due to high rates of birth defects such as craniofacial anomalies, ambiguous genitalia, and fetal death. ${ }^{11}$ HER2 positive cancers should not receive targeted therapy like Trastuzumab during pregnancy due to increased incidence of oligohydramnios/anhydramnios. ${ }^{2}$

Our case had huge tumor at diagnosis with axillary lymphadenopathy. Upfront surgery was not feasible in our case due to huge locally advanced tumor. We planned to start NAC before delivery to downstage the tumor. However, despite counselling by the treating team, patient and her relatives wished to deliver the baby first then only start NAC due to the fear of possible toxicity to the fetus. Though literature suggests acceptable short term risks of pre-eclampsia and intrauterine growth retardation $(I U G R)^{12}$, there is no robust evidence of long term effects of chemotherapy. As such, it is difficult for
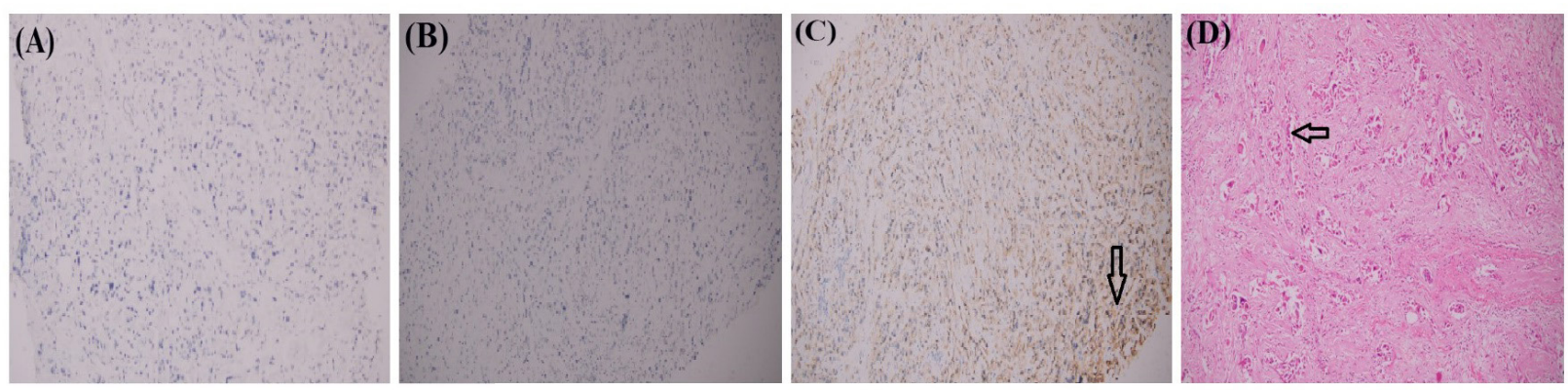

Figure 2. Immunohistochemistry of Trucut biopsy (100X) showing absence of expression of estrogen receptor $(A)$, progesterone receptor (B) and overexpression of HER2-neu (arrow) (C) Post mastectomy specimen with residual tumor (arrow) and desmoplasia (D) 
the treating team to convince the patients when they inquire about long term effects on the child.

One study shows HER2-enriched tumors have increased risk of death if NAC is delayed by more than 56 days $^{13}$ though it does not differentiate early and late groups by tumor size or focality. In our case, where the tumor was multifocal with huge size and patient started on NAC after 6 weeks, this delay of only 42 days could also be significant. Recent study showed NAC to result in more breast conservation without compromising on distant recurrence and survival ${ }^{14}$ and indeed is the preferred approach in advanced stage HER2enriched subtype in second and third trimesters. ${ }^{15}$

Our patient had HER2-enriched molecular subtype, which has aggressive biological behavior, with only partial response to chemotherapy, and was doing fine at 12 months follow up. Trastuzumab was advised postoperatively but patient could not afford it due to financial reasons. In a country like Nepal, management of such HER2 positive cases is a financial challenge ${ }^{16}$ and very few fortunate people can afford.

We do not have clear-cut data on PABC in Nepal. As the trend is increasing in West due to delayed pregnancy, we can infer similar trend in Nepal due to globalization. As such we have pressing need to keep records of such patients and follow them for their lifetime to make informed decisions.

\section{CONCLUSION}

PABC presents in advanced stage. Long term safety data would help patients make informed decision regarding the use and timely initiation of neoadjuvant chemotherapy in pregnancy. In a developing country like Nepal, access to universally recommended targeted therapy in breast cancer is limited by financial constraints.

\section{CONFLICT OF INTEREST}

None declared.

\section{CONSENT}

Written informed consent was obtained from the patient for publication of this report and accompanying images.

\section{REFERENCES}

1. Breast Cancer Treatment During Pregnancy. National Cancer Institute https://www.cancer.gov/ types/breast/hp/pregnancy-breast-treatmentpdq (1980).

2. Zagouri, F. et al. Trastuzumab administration during pregnancy: a systematic review and metaanalysis. Breast Cancer Res. Treat. 137, 349-357 (2013).

3. World Economic Outlook Database, International Monetary Fund. https://www.imf.org/external/ pubs/ft/weo/2016/01/weodata/weoselgr.aspx.

4. Agha, R. A. et al. The SCARE Statement: Consensus-based surgical case report guidelines. Int. J. Surg. Lond. Engl. 34, 180-186 (2016).

5. Moore, H. C. \& Foster, R. S. Breast cancer and pregnancy. Semin. Oncol. 27, 646-653 (2000).

6. Rugo, H. S. Management of breast cancer diagnosed during pregnancy. Curr. Treat. Options Oncol. 4, 165-173 (2003).

7. Clark, R. M. \& Chua, T. Breast cancer and pregnancy: the ultimate challenge. Clin. Oncol. R. Coll. Radiol. G. B. 1, 11-18 (1989).

8. Yang, W. T., Dryden, M. J., Gwyn, K., Whitman, G. J. \& Theriault, R. Imaging of breast cancer diagnosed and treated with chemotherapy during pregnancy. Radiology 239, 52-60 (2006).

9. Gradishar, W. J., Anderson, B. O. \& Abraham, J. NCCN Guidelines on Breast Cancer. Breast Cancer 212 (2018).

10. Koren, G. et al. Cancer Chemotherapy and Pregnancy. J. Obstet. Gynaecol. Can. 35, 263278 (2013)

11. Amant, F., Loibl, S., Neven, P. \& Calsteren, K. V. Breast cancer in pregnancy. The Lancet 379,570 579 (2012).

12. Cardonick, E. \& lacobucci, A. Use of chemotherapy during human pregnancy. Lancet Oncol. 5, 283291 (2004).

13. Sanford, R. A. et al. Impact of delayed neoadjuvant systemic chemotherapy on survival outcomes in breast cancer patients. J. Clin. Oncol. 34, 10381038 (2016).

14. Derks, M. G. M. \& Velde, C. J. H. van de. Neoadjuvant chemotherapy in breast cancer: more than just downsizing. Lancet Oncol. 19, 2-3 (2018).

15. Reyal, F., Hamy, A. \& Piccart, M. J. Neoadjuvant treatment: the future of patients with breast cancer. ESMO Open 3, (2018).

16. National Accounts - Analysis of Main Aggregates (AMA). https://unstats.un.org/unsd/snaama/ 